\title{
Briefly occlusive coronary anastomosis with tissue adhesive
}

\author{
Marc P. Buijsrogge, MD \\ Cees W. J. Verlaan \\ Paul F. Gründeman, MD, PhD \\ Cornelius Borst, MD, PhD
}

Objective: We assessed the feasibility of a facilitated, briefly occlusive, sutureless coronary anastomosis technique in which side-to-side preglued (octylcyanoacrylate adhesive) bounded walls were opened by a conventional arteriotomy.

Methods: In low-flow (prothrombotic milieu, $\leq 15 \mathrm{~mL} / \mathrm{min}, \mathrm{n}=8$ ) and high-flow (approximately $50 \mathrm{~mL} / \mathrm{min}, \mathrm{n}=8$ ) porcine model of off-pump internal thoracic artery-left anterior descending coronary artery bypass, the anastomoses were evaluated intraoperatively $(\mathrm{n}=16)$ and at 5 weeks $(\mathrm{n}=14$, with 2 evaluated at 2.5 weeks). The anastomoses were examined by flow measurement, angiography, and histologic studies.

Results: Coronary occlusion lasted a median of 1.6 minutes (15th-85th percentile 1.4-1.8 minutes), and anastomosis construction required a median of 5.5 minutes (15th-85th percentile 4.2-6.5 minutes). At 5 weeks all anastomoses were fully patent (FitzGibbon grade A), with an angiographic appearance similar to an end-to-side anastomotic configuration.

Conclusions: The briefly occlusive adhesive anastomosis technique proved to be feasible in off-pump bypass surgery in the pig even under prothrombotic low bypass graft flow conditions ( $\leq 15 \mathrm{~mL} / \mathrm{min}$ ). Further studies are warranted to investigate the applicability of this technique to endoscopic bypass surgery on the beating heart.

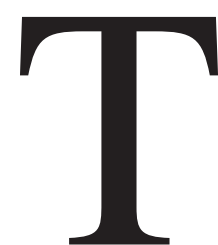

he use of adhesives in the coronary artery bypass grafting (CABG) anastomosis construction has the potential to accelerate and simplify this procedure. Tissue adhesives have been used for many years as alternative to tissue bonding by suture. ${ }^{1}$ In the construction of vascular anastomoses, however, the applicability of adhesives has been limited because of histotoxicity (aneurysm formation), adhesive entering the vessel lumen, and ineffectiveness of adhesives in cases of active bleeding.

The aim of this study was to assess the feasibility of a facilitated coronary anastomosis construction by a modified adhesive technique with a side-to-side pregluing approach initially described in $1964 .{ }^{2}$ Our objective was to assess the patency and vascular wall healing of the modified technique in off-pump porcine CABG under both low-flow (prothrombotic milieu) and high-flow conditions in the internal thoracic artery (ITA) graft. ${ }^{3}$

\section{Material and Methods \\ Animals}

Ten Dutch female Landrace pigs (weighing 70-90 kg) were used. The animals were fed a normal diet and received humane care in compliance with the "Guide for the Care and Use of Laboratory Animals" prepared by the Institute of Laboratory Animal Resources, National Research Council, and published by the National Academy Press. The protocol was approved by the animal experimentation committee of the Utrecht University. One day before surgery 

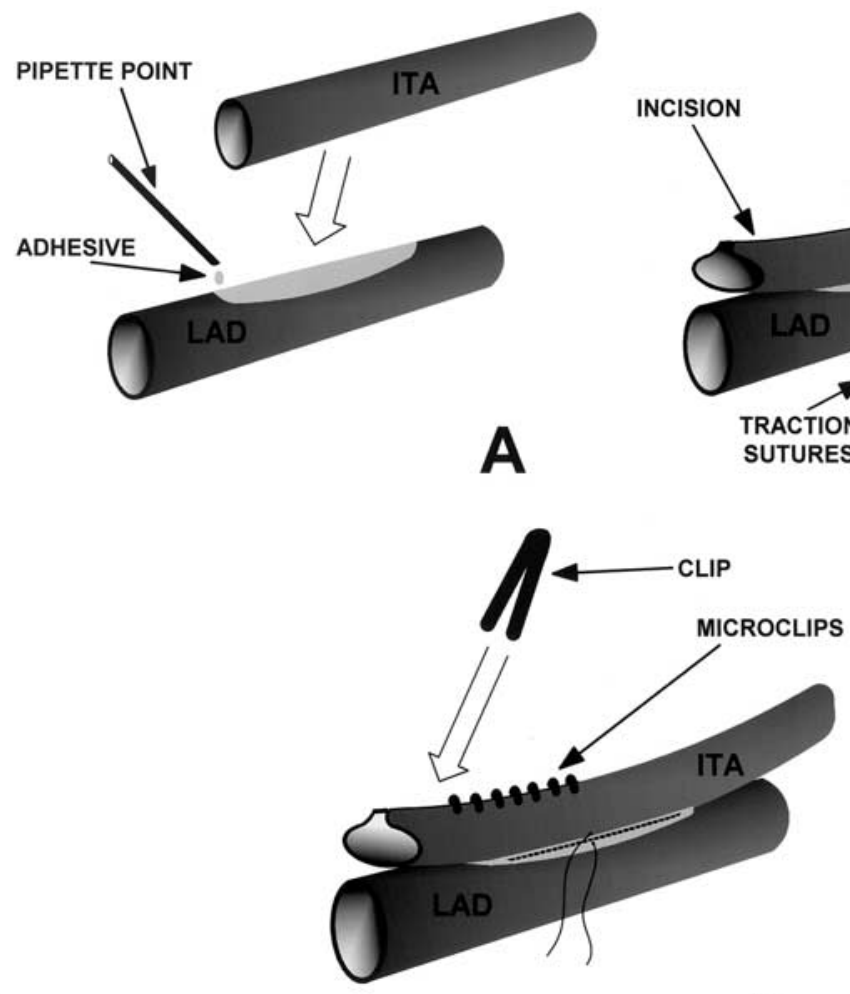

D
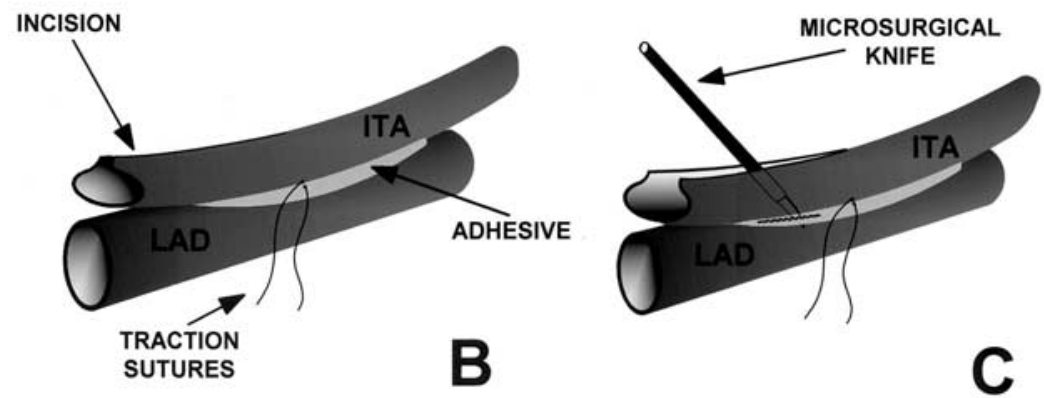

C

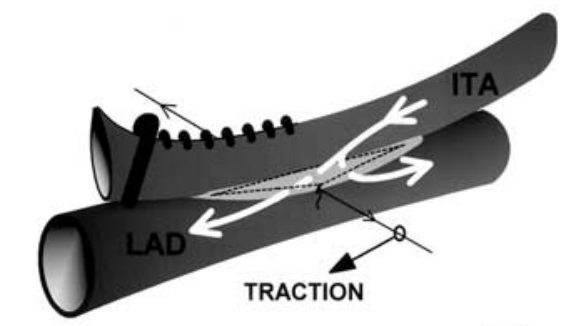

E

Figure 1. Anastomotic procedure. A, Adhesive (1.5 $\mu \mathrm{L})$ was applied to LAD by pipette point. B, After adhesive polymerization, top of ITA was incised to obtain adequate view of designated anastomotic site. Two traction sutures were placed at lateral sides of anastomosis. C, Arteriotomy was made through both vessel walls. D, Top incision was closed, and distal end of ITA was clipped. E, Two traction sutures were tied to epicardium to convert longitudinal arteriotomy into diamond-shape form.

all animals received $560 \mathrm{mg}$ acetylsalicylic acid orally, which was continued in a dose of $160 \mathrm{mg} / \mathrm{d}$ until they were killed.

\section{Anesthesia and Euthanasia}

The animals were anesthetized and humanely killed as described previously elsewhere. ${ }^{3}$

\section{Surgery}

Both left and right ITAs were harvested in a skeletonized fashion. After intravenous heparinization to obtain an activated clotting of twice the control value (determined at 10 and 60 minutes and every 30 minutes afterwards after injection until the end of procedure), both distal ITAs were dissected. The anastomotic segment of the left anterior descending coronary artery (LAD) was immobilized by the Octopus 3 Tissue Stabilizer (Medtronic, Inc, Minneapolis, Minn). The LAD outer diameter and ITA half circumference were measured with calipers.

\section{Anastomotic Procedure}

Under the microscope (magnification 6.25×, wild M680; Leica AG, Heerburg, Switzerland), the distal end of the ITA (cut at an angle of $90^{\circ}$ ) and the LAD were cleared of loose periadventitial tissue. Preischemic conditioning was used before coronary artery occlusion, which was performed with two microvascular Acland clamps (B-3V; S\&T Marketing LtD, Neuhausen, Switzerland).

With the use of a pipette (Proline Pipette; Biohit OY, Helsinki, Finland) and pipette point (200 $\mu \mathrm{L}$; Greiner GmbH, Frickenenhausen, Germany), about $1.5 \mu \mathrm{L}$ octylcyanoacrylate adhesive (Dermabond; Ethicon, Inc, Somerville, NJ) was applied to the coronary artery, after which the ITA was preglued onto the LAD over a distance of about $2 \mathrm{~cm}$ (Figure 1, A). After adhesive polymerization, the top of the graft was incised over a length of 1 $\mathrm{cm}$ (up to the designated location of the anastomosis). Subsequently two polypropylene 7-0 traction sutures (Ethicon) were placed at the lateral sides (at 3 and 9 o'clock positions) of the anastomosis (Figure 1, B).

After the pregluing procedure, an arteriotomy (through ITA and LAD vessel wall) was performed with a $15^{\circ}$ microsurgical knife (Sharpoint; Surgical Specialties Corporation, Reading, Pa), after which the LAD was occluded and the arteriotomy length was extended to $5 \mathrm{~mm}$ (Figure 1, C). After arteriotomy, the top incision of the ITA was closed (VCS microclips-small; US Surgical Corporation, Norwalk, Conn), and the distal end was clipped (medium Atraumaclip; Pilling, Inc, Fort Washington, Pa; Figure 1, 
TABLE 1. Operative data

$\left.\begin{array}{lcccc}\hline & \begin{array}{c}\text { Anastomosis } \\ \text { surgery time } \\ (\mathbf{m i n})\end{array} & \begin{array}{c}\text { Coronary } \\ \text { occlusion } \\ \text { time }(\mathbf{m i n})\end{array} & \begin{array}{c}\text { ITA inner LAD inner } \\ \text { diameter } \\ (\mathbf{m m})\end{array} & \begin{array}{c}\text { diameter } \\ (\mathbf{m m})\end{array} \\ \hline \text { Low flow } & 5.2 \pm 1.2 & 1.5 \pm 0.3 & 1.6 \pm 0.2 & 1.9 \pm 0.3 \\ \text { High flow } & 5.5 \pm 1.1 & 1.7 \pm 0.2 & 1.6 \pm 0.2 & 2.5 \pm 0.3\end{array}\right\} P=.003$

Data are presented as mean \pm SD except where noted $(n=16$ anastomoses in 8 animals).

*Diameter of LAD was less in the more distally positioned, low-flow anastomoses. $^{3}$

$D)$, after which the graft flow was restored. The two traction sutures at the lateral sides of the anastomosis were tied to the epicardium to convert the longitudinal arteriotomy into a diamond shape (Figure 1,E).

Two anastomoses were constructed in each animal, a distal low-flow $(\leq 15 \mathrm{~mL} / \mathrm{min}$ ) right ITA-LAD anastomosis and a proximal high-flow (about $50 \mathrm{~mL} / \mathrm{min}$ ) left ITA-LAD anastomosis. ${ }^{3}$ All anastomoses were performed by a single investigator (M.P.B.). The animals were evaluated intraoperatively and at 5 weeks.

\section{Intraoperative and Postoperative Measurements}

During the operation and when the animals were killed, ITA flow and coronary peak hyperemic response were measured and recorded with a calibrated transit time flow probe (3S) connected to a flow meter (model T208; Transonic systems, Inc, Ithaca, NY) at a mean blood pressure of $70 \mathrm{~mm} \mathrm{Hg}$, as described previously elsewhere. ${ }^{3}$ Before chest closure, the ITA flow was monitored continuously for as long as 2 hours.

\section{Angiography}

After the animals were killed the anastomoses were visualized by ITA angiography (C-arm BV27; Philips, Eindhoven, The Netherlands) at $80 \mathrm{~mm} \mathrm{Hg}$ and graded by an independent observer according to the FitzGibbon criteria.

\section{Histologic Studies}

After angiography the anastomoses were perfused for 30 minutes with $4 \%$ formalin at $80 \mathrm{~mm} \mathrm{Hg}$. Subsequently, anastomotic and reference segments (proximal LAD and ITAs) were sectioned and stained as described previously elsewhere. ${ }^{4}$

\section{Statistical Analysis}

Data are presented as mean $\pm \mathrm{SD}$ or as median and 15th through 85 th percentile. Repeated measurement analysis of variance by general linear model was used to compare means with the SPSS software package (SPSS, Inc, Chicago, Ill).

\section{Results}

\section{Surgery}

The operative data are given in Table 1. Pregluing of the ITA onto the LAD required $3.4 \pm 1.0$ minutes (from adhesive application until completion of polymerization). Be-
TABLE 2. Intraoperative and postoperative measurements

\begin{tabular}{|c|c|c|}
\hline & Low flow & High flow \\
\hline \multicolumn{3}{|c|}{ ITA graft flow (mL/min) } \\
\hline Intraoperative & $11 \pm 2$ & $50 \pm 16$ \\
\hline $2 \mathrm{~h}$ & $11 \pm 3$ & $52 \pm 16$ \\
\hline 5 wk & $10 \pm 5$ & $42 \pm 11^{*}$ \\
\hline \multicolumn{3}{|c|}{$\begin{array}{l}\text { Coronary peak hyperemic response } \\
\text { (peak/baseline flow ratio) }\end{array}$} \\
\hline Intraoperative & $5.2 \pm 1.2$ & $2.7 \pm 0.6$ \\
\hline $5 \mathrm{wk}$ & $5.6 \pm 1.8 \dagger$ & $4.2 \pm 1.1^{*}$ \\
\hline
\end{tabular}

Data are presented as mean \pm SD ( $n=14$ anastomoses in 7 animals). Intraoperative measurements were made after stabilization of flow after completion of anastomosis. $P=.001$ for difference between low-flow and high-flow anastomoses at intraoperative period, 2 hours, and 5 weeks, as intended by the low-flow and high-flow model. ${ }^{3}$

*Because of imperfect occlusion of main stem, 1 animal was excluded from flow analysis.

tBecause of imperfect occlusion of midsegment LAD, 1 animal was excluded from flow analysis.

cause of an insufficient amount of adhesive applied to the LAD in 1 animal, pregluing had to be redone after the misapplied adhesive was peeled off the LAD. Arteriotomy and ITA incision closure (from microclip placement until clipping of the distal end of the ITA) required $0.5 \pm 0.1$ minutes and $0.9 \pm 0.2$ minutes, respectively. In 1 animal closure of the ITA incision lasted 3.0 minutes as a result of malfunctioning of the microclip stapler, and that time measurement was discarded.

Immediate full hemostasis was obtained in 11 of 16 anastomoses. In 1 case, two 8-0 stitches had to be placed at the lateral side of the anastomosis after 1 minute to obtain complete hemostasis. In 4 cases the ITA incision closed by microclips required an extra hemostatic stitch. The activated clotting times before heparin administration, during the anastomotic procedure, and at 270 minutes after heparinization were $106 \pm 12,235 \pm 33$ and $156 \pm 14$ seconds, respectively.

\section{Follow-up}

The scheduled 5 weeks of follow-up was complete for 7 animals. One animal suddenly died 5 hours after the operation of mechanically induced ventricular fibrillation caused by displacement of the animal. Two animals died of saddletype pulmonary embolism on days 2 and 19. In all 3 of these animals the anastomoses, examined angiographically and histologically, were without flaws or mural thrombus. The animals that died 5 hours and 2 days after the operation were discarded from analysis. During the 5 weeks of follow-up the weight of the 7 remaining animals was stable $(77 \pm 12 \mathrm{~kg}$ and $76 \pm 11 \mathrm{~kg})$.

\section{Intraoperative and Postoperative Measurements} ITA flow measurements are given in Table 2. No (cyclic) 


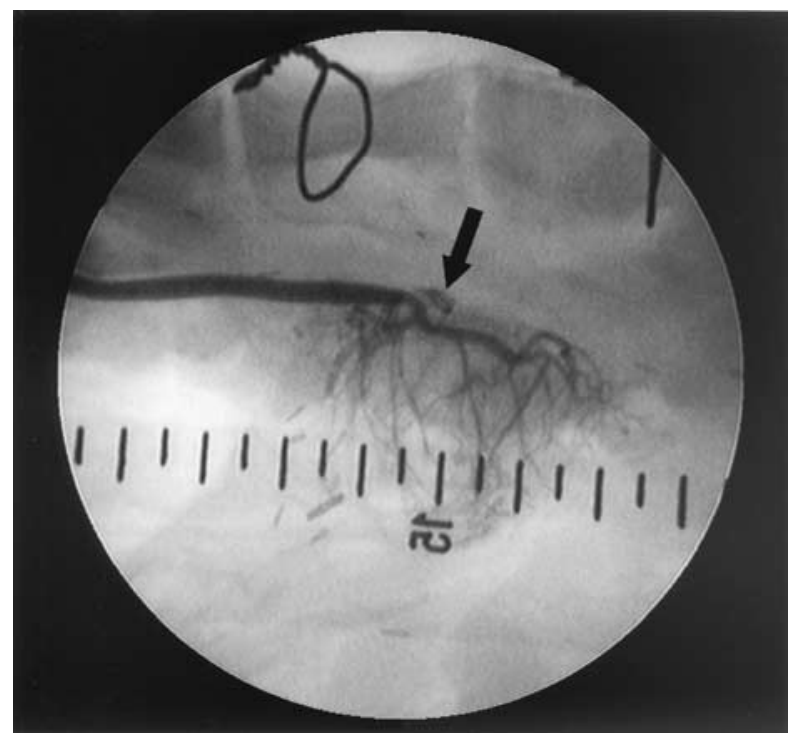

Figure 2. Representative angiogram of low-flow adhesive anastomosis at 5 weeks after operation demonstrating angiographic appearance similar to end-to-side anastomosis. Note clip placed at distal end of ITA (black arrow).

flow reductions were observed during 2 hours of flow monitoring after anastomosis construction.

\section{Angiography}

When the animals were killed, all anastomoses met FitzGibbon grade A criteria (Figure 2). No anastomotic narrowing or aneurysm formation was observed.

\section{Histologic Studies}

At 5 weeks the intraluminally exposed rim of the inverted vessel walls (media and small rim of adventitia of LAD and ITA because of the method of preglued bounded vessel walls opening) led to a slight excess of vessel wall graft material at the anastomotic orifice, which was covered by neointimal repair tissue (Figure 3). No dehiscence was seen, but fibroblastic (repair) tissue was formed in between the glued vessel walls (Figure 4). Toward the toe of the anastomosis (the clipped distal end of the ITA), the neointimal repair tissue reorganized the lumen of the ITA graft (Figure 4).

Medial necrosis. Full-thickness media necrosis was recorded near all microclip elements at the top incision of the ITA. No medial necrosis (coronary or ITA) was observed near the adhesive.

Adhesive. At 5 weeks a focal acute (polymorphonuclear cells and macrophages $>100$ inflammatory cells per field, magnification $1000 \times$ ) and limited chronic inflammatory cell reaction (foreign body giant cells occasionally seen) was confined to the (adventitial) adhesive application site. In 10 of 14 anastomoses, however, scattered acute inflam-

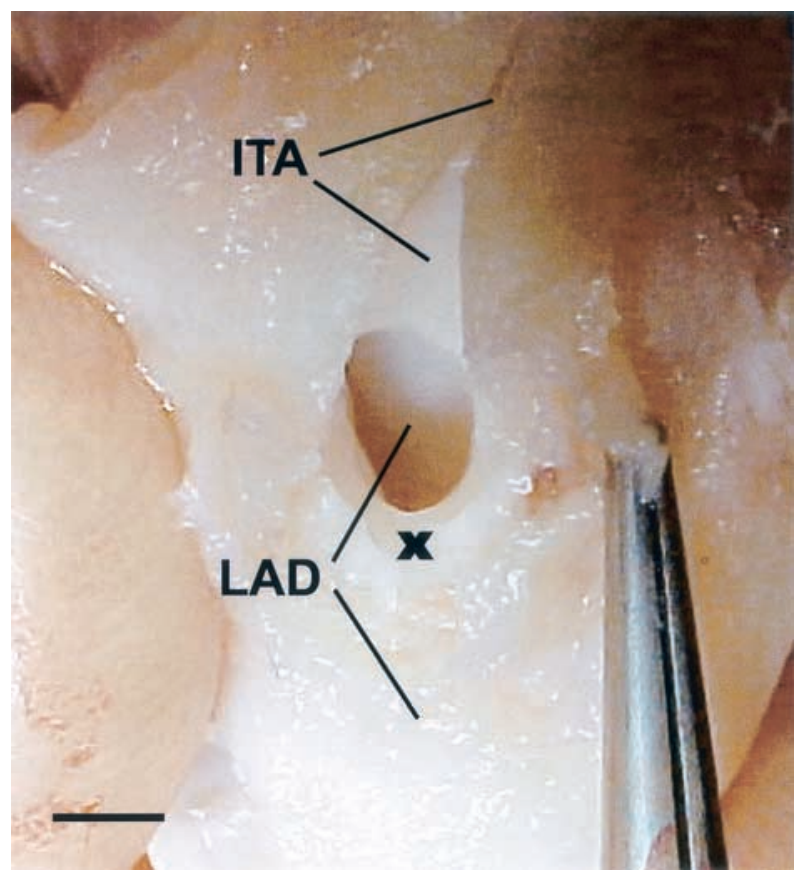

Figure 3. Gross view from the top of bypass graft into adhesive anastomosis after excision at 5 weeks. Black $x$ indicates clipped distal end of ITA.

matory cells ( $\leq 100$ cells per field) were observed in the outer media of the ITA. No extensive or full-thickness media inflammation was seen.

\section{Discussion}

The principal results of this study were as follows: (1) All sutureless anastomoses were fully patent (FitzGibbon grade A) without aneurysm formation. (2) The adhesive technique enabled briefly occlusive (median 1.6 minutes, 15th-85th percentile 1.4-1.8 minutes), simple, and fast (median 5.5 minutes, 15 th- 85 th percentile $4.2-6.5$ minutes) coronary artery anastomosis construction.

\section{Sutureless Adhesive Anastomosis}

The use of tissue adhesives to facilitate vascular anastomosis construction is theoretically attractive. In general, adhesives are capable of simplifying tissue bonding processes (single application instead of multiple complex suture placement) without large or bulky instrumentation tools, which makes them suitable for application in reduced working space or difficult to access (coronary) target areas. 5,6 However, their use in vascular anastomosis construction has been largely disappointing because of problems with their histotoxicity, adhesive becoming exposed intraluminally, and ineffectiveness in the presence of blood. 7,8

To address at least part of these limitations, a modified distal sutureless anastomosis technique was used in which 
the graft was preglued to the coronary artery, as originally proposed by Yahr and colleagues. ${ }^{2}$ With an incision at the top of the distal end of the ITA, an arteriotomy was performed after which both the incision and distal end of the graft were closed (Figure 1). A new homolog, octylcyanoacrylate, currently approved by the Food and Drug Administration for topical wound closure, was selected because of its strong, flexible bond and relatively low histotoxicity. ${ }^{4,9}$

Without intraluminal foreign body exposure and without use of any cumbersome application tools, we were able to perform a simple and fast distal anastomosis that required a median of only 1.6 minutes (15th-85th percentile 1.4-1.8 minutes) of coronary occlusion. After 5 weeks all sutureless anastomoses, even under prothrombotic low bypass graft flow conditions of less than $15 \mathrm{~mL} / \mathrm{min}$, were both angiographically (FitzGibbon grade A) and hemodynamically (peak hyperemic flow response $4.9 \pm 1.6$ ) fully patent. After 5 weeks in the porcine model (which corresponds to 15 to 30 weeks of wall healing in the stented human coronary $\operatorname{artery}{ }^{10}$ ), neointimal repair tissue formation was formed that covered the inverted anastomotic vessel walls and reorganized the side-to-side approach into an end-toside anastomotic configuration (Figures 2 and 3).

Limitations of the sutureless adhesive procedure are, first, the ineffectiveness of octylcyanoacrylate adhesive in the presence of blood. Second, the relatively large dose of $1.5 \mu \mathrm{L}$ adhesive, which is not yet approved for internal use, occasionally caused an acute inflammatory reaction in the outer media of the ITA, although this was without detrimental effect at 5 weeks. Third, the arteriotomy, when performed by a conventional microknife, was a delicate and time-consuming procedure (the incision on the top of the ITA and subsequent closure took $1.2 \pm 0.3$ minutes) and led, at least during the operation, to an excess of vessel wall graft at the anastomotic orifice.

In a more highly developed fashion (in which a punch, introduced into the distal end of the ITA, could be used for both arteriotomy and vessel wall approximation), we believe that this sutureless anastomosis technique with either a highly effective chemical or biologic adhesive would enable simple, fast, and potentially long-term effective (no intraluminal bonding element exposure) coronary anastomosis construction in limited access CABG procedures. So far, however, the feasibility and long-term effect (histotoxicity and anastomotic quality) of this study's sutureless anastomosis technique remain to be established in atherosclerotic human coronary arteries.

\section{Conclusions}

The adhesive side-to-side distal anastomosis technique, modified from the description of Yahr and colleagues, ${ }^{2}$ proved to be feasible in porcine off-pump CABG, even under prothrombotic low bypass graft flow conditions $(\leq 15$

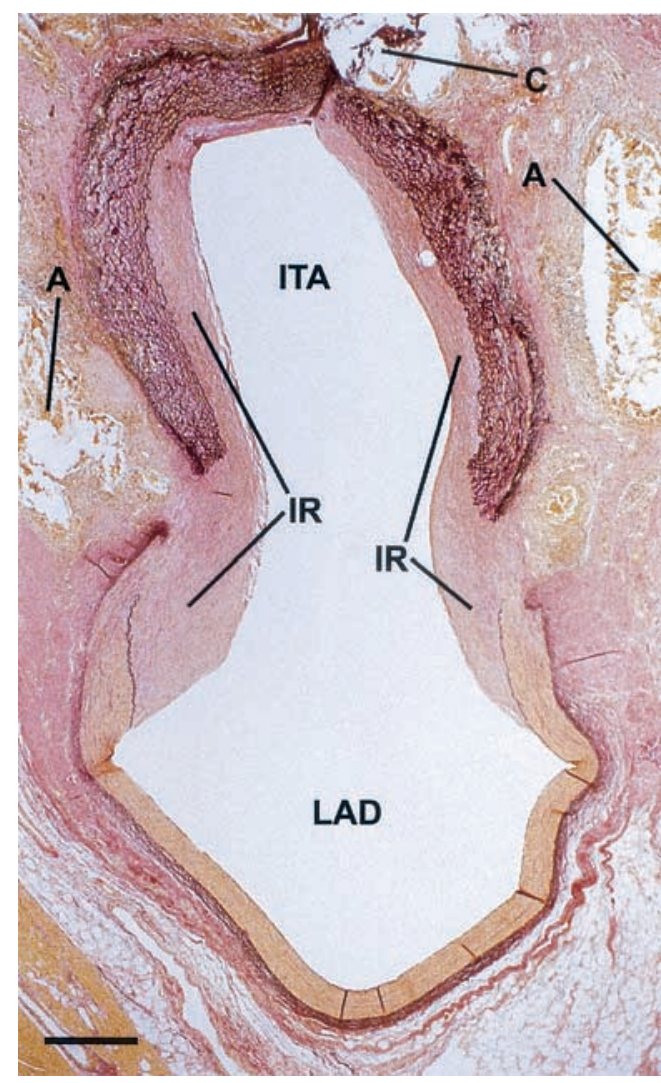

Figure 4. Transverse cross-section of segment toward toe of low-flow adhesive anastomosis at 5 weeks after operation (not pressure fixed). Note rim of (former) inverted vessel walls covered by neointimal repair tissue (IR) and reorganized lumen of ITA graft. $\boldsymbol{A}$, Adhesive; $\boldsymbol{C}$, position of clip (top incision closure) after its removal (elastin van Gieson stain; bar represents $250 \mu \mathrm{m}$ ).

$\mathrm{mL} / \mathrm{min}$ ). The technique enabled briefly occlusive (median 1.6 minutes, 15 th-85th percentile 1.4-1.8 minutes) and fast (median 5.5 minutes, 15th-85th percentile 4.2-6.5 minutes) anastomosis construction. Further studies are warranted to investigate the applicability of this technique in endoscopic CABG on the beating heart.

We acknowledge the constructive contributions of A. R. T. Donders, $\mathrm{PhD}$, from the Center of Biostatistics, University of Utrecht, and M. H. P. van Rijen, BSc, M. Schurink, and colleagues from the Utrecht University Central Animal Facilities.

\section{References}

1. Lerner R, Binur NS. Current research review: current status of surgical adhesives. J Surg Res. 1990;48:165-81.

2. Yahr WZ, Strully KJ, Hurwitt ES. Non-occlusive small arterial anastomosis with a neodymium laser. Surg Forum. 1964;15:224-5.

3. Buijsrogge MP, Gründeman PF, Verlaan $\mathrm{CW}$, Borst C. Unconventional vessel wall apposition in off-pump porcine coronary bypass grafting: low versus high graft flow. J Thorac Cardiovasc Surg. 2002;123:341-7. 
4. Buijsrogge MP, Scheltes JS, Heikens M, Grundeman PF, Pistecky PV, Borst C. Sutureless coronary anastomosis with an anastomotic device and tissue adhesive in off-pump porcine bypass grafting. J Thorac Cardiovasc Surg. 2002;123:788-94.

5. Aksik IA, Kikut RP, Apshkalne DL. Extraintracranial anastomosis performed by means of biological gluing materials: experimental and clinical study. Microsurgery. 1986;7:2-8.

6. Gundry SR, Black K, Izutani H. Sutureless coronary artery bypass with biologic glued anastomoses: preliminary in and in vitro results. J Thorac Cardiovasc Surg. 2000;120:473-7.

7. Toriumi DM, Raslan WF, Friedman M, Tardy ME. Histotoxicity of cyanoacrylate tissue adhesives. Arch Otolaryngol Head Neck Surg. 1990; $116: 546-50$

8. Takenaka H, Esato K, Ohara M, Zempo N. Sutureless anastomosis of blood vessels using cyanoacrylate adhesives. Surg Today. 1992;22: 46-54.

9. Ang ES, Tan KC, Tan LH, Ng RT, Song IC. 2-Octylcyanoacrylateassisted microvascular anastomosis: comparison with a conventional suture technique in rat femoral arteries. J Reconstr Microsurg. 2001; 17:193-201.

10. Fischell TA, Virmani R. Intracoronary brachytherapy in the porcine model: a different animal. Circulation. 2001;104:2388-90. 